\title{
Anisotropic Diffusion of Light in a Strongly Scattering Material
}

\author{
Patrick M. Johnson, ${ }^{1,2, *}$ Boris P. J. Bret, ${ }^{1, \dagger}$ Jaime Gómez Rivas, ${ }^{1}$ John J. Kelly, ${ }^{2}$ and Ad Lagendijk ${ }^{1, \dagger}$ \\ ${ }^{1}$ Van der Waals-Zeeman Instituut, Universiteit van Amsterdam, 1018 XE Amsterdam, The Netherlands \\ ${ }^{2}$ Debye Insitituut, Universiteit Utrecht, P.O. Box 80000, 3508 TA Utrecht, The Netherlands
}

(Received 20 May 2002; published 20 November 2002)

\begin{abstract}
Light transport in a strongly scattering, strongly anisotropic material is studied experimentally using both static and time-resolved techniques. Both the static and the dynamic results are well characterized by a diffusion equation with an anisotropic diffusion tensor and a scalar absorption term. Light diffuses 4.0 times faster along the uniaxial axis of the material compared with diffusion in the orthogonal directions.
\end{abstract}

DOI: 10.1103/PhysRevLett.89.243901

PACS numbers: 42.25.Dd, 78.30.Ly, 78.55.Mb

The study of light transport in strongly scattering materials underlies such diverse subjects as Anderson localization of light [1], optical imaging in biological systems [2], and diffusing wave spectroscopy [3]. In these and other types of studies, a clear understanding of the diffusive regime, in which light transport is well modeled by the diffusion equation, is essential for progress. It is somewhat surprising then that little experimental work has been done on strongly scattering systems in which statistical anisotropy exists. This lack of study is due in part to the difficulty of creating a strongly scattering random optically anisotropic system in a controlled way. Strongly scattering powders of high dielectric materials [4] are statistically isotropic over length scales much larger than the particle size, while single domain nematic liquid crystal systems, which do show anisotropic optical diffusion [5-8], are comparatively weakly scattering and weakly anisotropic. Recently, macroporous gallium phosphide has been shown to be the strongest random scatterer of light in the visible regime with weak absorption $[9,10]$. In this Letter, we show that this material offers a unique opportunity to study strong scattering in the presence of statistical anisotropy. By controlling the etching conditions, strongly scattering samples with a high degree of optical anisotropy can be created. The geometry of these samples allow both static and time-resolved measurements to be performed, the results of which consistently describe strongly anisotropic diffusion of light.

Previous studies have shown that light may propagate both diffusively and anisotropically in nematic liquid crystals [5-8]. These systems are comparatively weakly scattering, with a mean free path $l$ of hundreds of microns [11] and a weakly anisotropic diffusion tensor $[5,6]$. Both the anisotropic scattering centers and an anisotropic index of refraction affect the transport properties of light $[7,8]$.

Macroporous gallium phosphide offers an entirely different material in which to study light propagation in the presence of anisotropy. The constituent materials, air and gallium phosphide, are both optically isotropic in the bulk, so the geometry of the material alone introduces the anisotropy. Under appropriate fabrication conditions, the material can be made to be extremely strongly scattering, with mean free paths as low as $l=0.3 \mu \mathrm{m}$ [10], i.e., on the order of the wavelength of light where Anderson localization of light is predicted to occur [1]. We show that samples with highly correlated anisotropy over centimeter length scales can be easily fabricated, using well known and commonly available materials and techniques. The degree of anisotropy in these samples can easily be tuned from isotropic or weakly anisotropic to the strongly anisotropic samples that we investigate here. This degree of control is particularly interesting because Anderson localization of light is theoretically predicted, in some cases, to be more easily achieved in the presence of statistical anisotropy [12]. A simple interpretation of this theory is that anisotropy makes the system effectively lower dimensional which helps localization to occur. Reference [12] describes structures that are intermediate between 1D and 3D systems. Our system can be described as intermediate between 2D and 3D. Such materials raise the prospect for empirically probing the effect of anisotropy on Anderson localization of light in a new geometry.

Porous semiconductors are fabricated by electrochemically etching single crystals in an acid solution. The pores in electrochemically etched gallium phosphide branch repeatedly during the etching process to create disorder in three dimensions. We have chosen etching conditions for which the samples are highly anisotropic while still showing disorder in three dimensions. Such materials are expected to show strong scattering, random light propagation, and a uniaxial optical anisotropy along the direction of etching. We have also devised an etching technique that aligns the direction of the pores parallel to the surface of the wafer. Such a geometry is essential for studying anisotropic light propagation in a uniaxial sample because it allows for a direct comparison of the directiondependent diffusion rates through imaging the surface of the sample. With careful surface preparation, it is surprisingly easy to etch in this fashion.

Our approach is to etch the entire thickness of gallium phosphide wafers starting from a thin edge and 
proceeding millimeters in, while controlling the orientation of the anisotropy [Fig. 1(a)]. N-type gallium phosphide [100] wafers were first coated with $\sim 300 \mathrm{~nm}$ amorphous silicon-nitride layers via plasma-enhanced chemical vapor deposition to prevent etching at the outside surfaces of the wafer. The layer is transparent and is thin enough to not appreciably change the thickness of the wafer $(L=310 \mu \mathrm{m})$. However, it is thick enough to seal the gallium phosphide from the sulfuric acid. The coated sample was then cleaved along a [100] plane and dipped into a $0.5 \mathrm{M}$ sulfuric acid solution with a potential applied along the long axis of the sample [Fig. 1(a)]. The voltage was applied by attaching an electrode to the top of the wafer, $\sim 1.5 \mathrm{~cm}$ from the opposite immersed edged. The electrochemical etching begins at the immersed edge of the wafer and progresses parallel to the wafer surface. The pores grow at a rate of roughly $0.5 \mathrm{~mm} /$ day, allowing for samples several $\mathrm{mm}$ wide to be created in a reasonable time. Strongly anisotropic pores were achieved by etching wafers with a doping concentration $7 \times 10^{17} \mathrm{~cm}^{-3}$ and a potential of $10.5 \mathrm{~V}$ [13]. Test samples were cleaved to obtain scanning electron micrograph (SEM) cross sections of the etching both parallel and perpendicular to the etching direction [Fig. 1(b), top and bottom, respectively]. Analysis of these images show that the etching proceeds in a dendritic fashion with pore diameters of $(70 \pm 30) \mathrm{nm}$. The characteristic length of the pores is several times its diameter, though the exact aspect ratio is difficult to determine because the cleavages often do not produce a smooth interior surface to examine. The etching proceeds uniformly up the sample with a planar front that extends to the inner edge of the SiN. Images of several different regions show the pore structure to be homogeneous throughout the sample, even when comparing regions several $\mathrm{mm}$ (i.e., several days) apart. Thus, a)

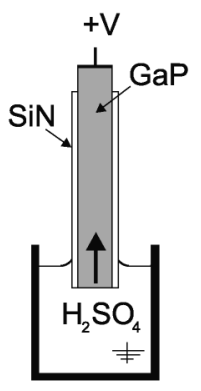

b)

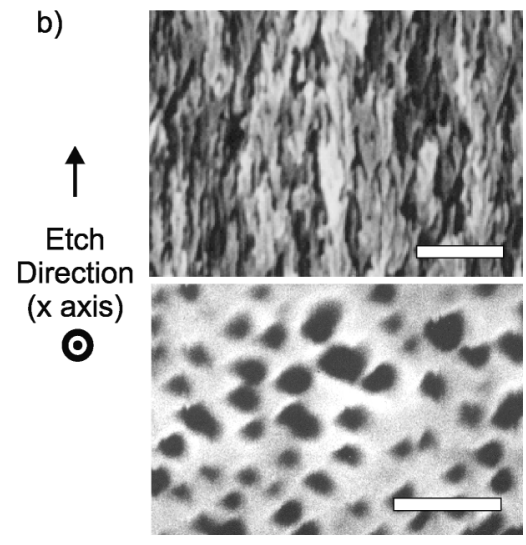

FIG. 1. (a) Cartoon of the etching setup for anisotropic etching. Because the silicon nitride layer prevents etching at the polished surface of the wafer, pores grow from the bottom edge up. (b) SEM image of the pores. Both parallel (top image, scale bar $=1 \mu \mathrm{m}$ ) and perpendicular (bottom image, scale bar $=$ $300 \mathrm{~nm}$ ) cross sections with respect to the etch direction (the $x$ axis) give a sense of the three-dimensional pore structure. the process should allow for even larger samples to be grown.

The most direct test for optical anisotropy is to create a pointlike source on one side of the sample and image the outgoing light on the opposite side. This was carried out with a solid-state pulsed laser source at $634 \mathrm{~nm}$ with an average power of $0.5 \mathrm{~mW}$ and a pulse duration of $\sim 100 \mathrm{ps}$. The source was focused to a $10 \mu \mathrm{m}$ spot. The image was recorded with a CCD camera [Fig. 2(a)]. The resulting image [Fig. 2(b)] shows elliptical isointensity contours with the long axis of the ellipse along the etching direction (the $x$ axis). Rescaling the $x$ axis of this image by a factor of 2.0 reduces these contours to circles [Fig. 2(b)]. This image shows already that light propagation in the sample is highly anisotropic.

To fully characterize the light transport in the sample, a second, time-resolved, setup was used (Fig. 3, top). The same 100 ps source was used, with the output side of the sample imaged and magnified through a microscope objective onto an aperture. By displacing the objective parallel to the sample surface, the image of the probed region of the sample surface could be translated across the aperture. The aperture was imaged onto the entrance of a monochrometer with a photomultiplier tube. The size of the aperture yielded a $50 \mu \mathrm{m}$ resolution for the displacement position. To resolve the signal in time, a time-toamplitude converter was used, triggered by a reference detector at the source output.

The resulting time-resolved signal is shown in the plot in Fig. 3. Shown are the results for three different displacements across the sample, $y=[(0,120$, and 240) \pm 10] $\mu \mathrm{m}$. Displacements of up to $480 \mu \mathrm{m}$ were measured in both the $x$ and $y$ directions where the $x$ and $y$ axes are shown in Fig. 2(b). The data show a peak followed by exponential decay characteristic of diffusion [1]. We find the exponential decay time to be independent of the

a)

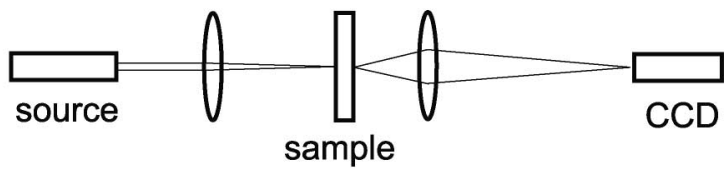

b)

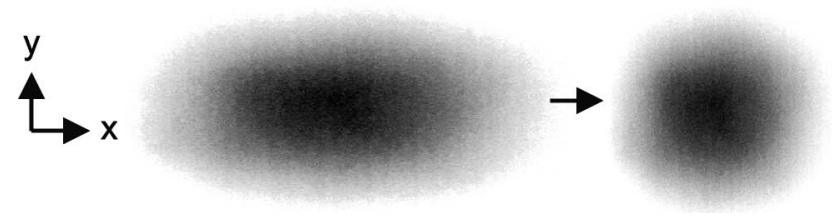

FIG. 2. (a) Cartoon of the static imaging setup. (b) The output image from the sample. The width of the wide distribution on the left is approximately $1.5 \mathrm{~mm}$. For the sake of presentation, the image has been inverted so that the darkest regions are those of highest intensity. On the right, the same image is shown with the $x$ axis rescaled by a factor of 2.0. This result demonstrates strongly anisotropic diffusion of light. 


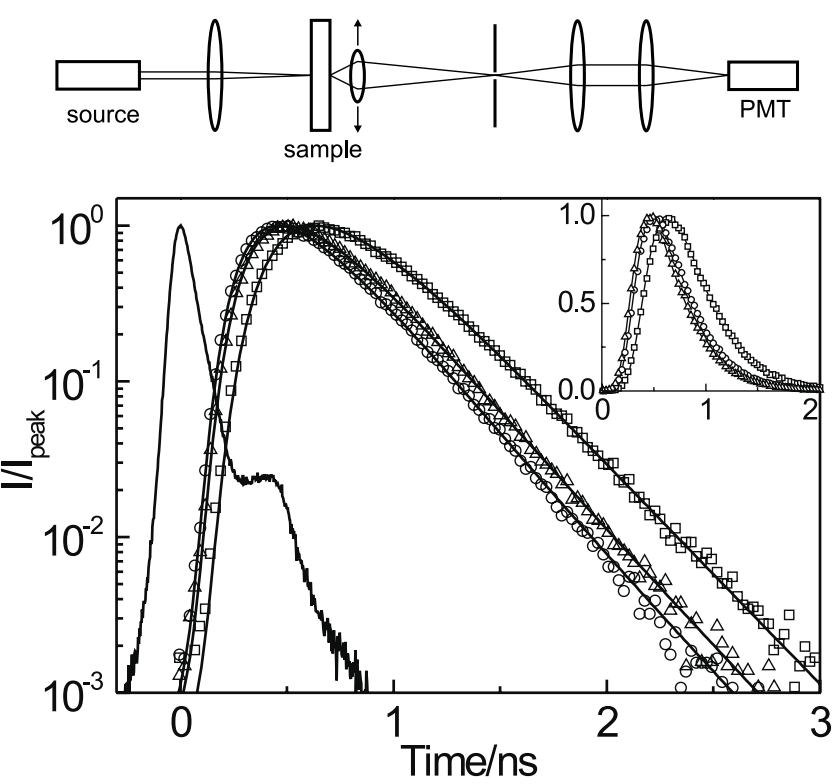

FIG. 3. Above: Cartoon of the time-resolved diffusion setup. Below: Normalized output intensity as a function of time for three different translations in the $y$ direction; $y=(0 \pm 10) \mu \mathrm{m}$ (circles), $y=(120 \pm 10) \mu \mathrm{m}$ (triangles), and (240 \pm 10$) \mu \mathrm{m}$ (squares). The solid lines give the fits to the anisotropic diffusion model for the same set of parameters at the three different displacements. The solid line on the left is the input pulse. The inset gives the same results with a linear vertical axis. The excellent agreement with the model shows that light propagates diffusively in these samples.

displacement, though the peak position shifts roughly in proportion to the square of the displacement. This shift is particularly evident in the inset of Fig. 3. The shift in the peak position is indicative of the increased average transmission time $t_{\mathrm{avg}}=\int I(t) t d t / \int I(t) d t$, a useful measure of the diffusion parameters. This has been calculated and plotted in Fig. 4. The anomalous point in this figure, the open triangle, shows the influence of the sample edge at $y=480 \mu \mathrm{m}$. Using the same setup, the static measurement can also be repeated, the results of which are shown in Fig. 5. As we now show, all of these results are in complete agreement with strongly anisotropic diffusion in this strongly scattering sample.

The diffusion model begins with the anisotropic diffusion equation with absorption:

$$
S+\frac{\partial I}{\partial t}=\left(D_{x x} \frac{\partial^{2}}{\partial x^{2}}+D_{y y} \frac{\partial^{2}}{\partial y^{2}}+D_{z z} \frac{\partial^{2}}{\partial z^{2}}\right) I-\frac{1}{\tau} I,
$$

where $I=I(r, t)$ is the intensity as a function of position and time, $S=S(r, t)$ is the source, $D_{x x}, D_{y y}$, and $D_{z z}$ are the diagonal components of the diffusion tensor, and $\tau$ is the absorption time. Given the uniaxial symmetry of the sample along the etching direction, off-diagonal elements of $D$ are not needed. The $z$ axis is parallel to the wafer normal. The tightly focused source beam may be approximated by a delta function $S \propto \delta(x) \delta(y) \delta(z+$

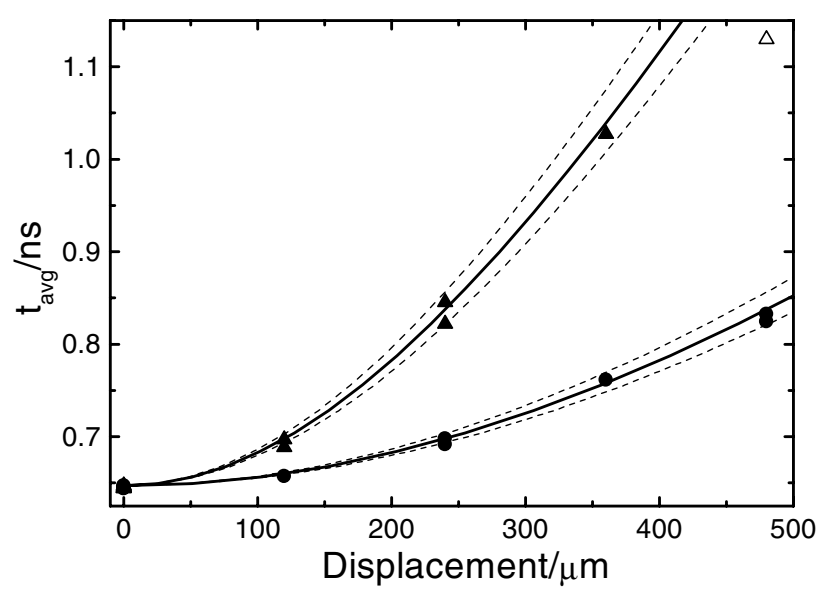

FIG. 4. The average transmission time $t_{\mathrm{avg}}$ vs displacement from center in the $x$ (circles) and $y$ (triangles) directions. The solid lines are fits to the diffusion model described in the text. The dashed lines give the curves at $\delta D_{x x, y y}=10 \%$. The strong anisotropy in $t_{\text {avg }}$ is consistent with the strong anisotropy in the diffusion tensor $\left(D_{x x} / D_{y y}=4.0\right)$.

$\left.z_{s}\right) \delta(t)$, where $z_{s}$ is the penetration depth of the incident beam. The mean free path for these samples has been previously measured at $l \approx 1 \mu \mathrm{m}$ [9] and we assume $z_{s} \approx$ $l$. Note that rescaling the $x$ and $y$ values by $1 / \sqrt{D_{z z} / D_{x x}}$ and $1 / \sqrt{D_{z z} / D_{y y}}$, respectively, reduces Eq. (1) to the isotropic diffusion equation, with the source term scaled by a constant factor. This explains our observed scaling result in Figs. 2 and 5 without further calculation and gives meaning to the measured scaling value 2.0, namely, $2.0=\sqrt{D_{x x} / D_{y y}}$ or $D_{x x}=4.0 D_{y y}$. The diffusion equation (1) can be solved analytically for both the timeresolved and stationary $(\partial I / \partial t=0)$ cases.

The time-resolved result was first fit to the $x=y=0$ position, where only $D_{z z}$ and $\tau$ remain as independent

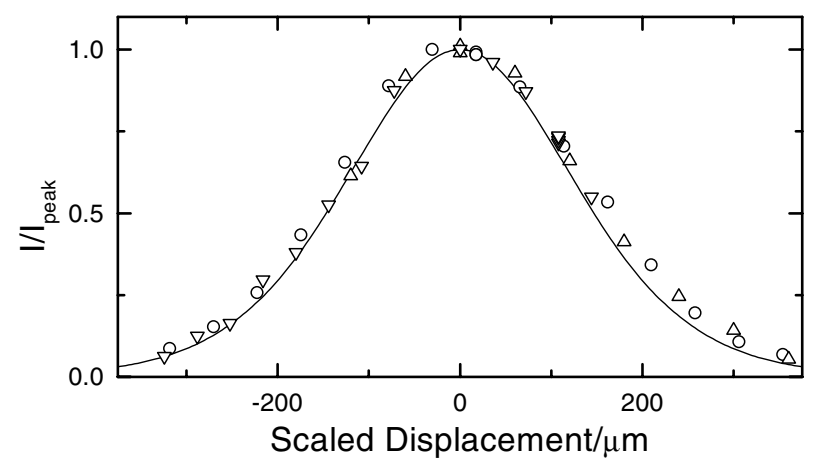

FIG. 5. The intensity vs displacement from center in the $x$ (triangles) and $y$ (circles) directions. The displacement in the $x$ direction has been rescaled by $\sqrt{D_{x x} / D_{y y}}=2.0$. Because of the wide intensity distribution along the $x$ axis, measurements for two different source positions were required to avoid edge effects. These are denoted by the two orientations of the triangles. The solid line is the result for the diffusion model using the parameters obtained from the time-resolved experiments. 
parameters. Fitting to the data set in Fig. 3 yields $D_{z z}=$ $(14.2 \pm 0.1) \mathrm{m}^{2} / \mathrm{s}$ and $\tau=(0.59 \pm 0.02) \mathrm{ns}$ where the error bars give the variance of the parameters. With these parameters fixed, the data was then fit to the displacements in both the $x$ and $y$ directions, yielding values of $D_{y y}=(14 \pm 2) \mathrm{m}^{2} / \mathrm{s}$ and $D_{x x}=(58 \pm 5) \mathrm{m}^{2} / \mathrm{s}$ where the uncertainty gives the range of values measured for different regions of the sample. The fits for the $y$ direction are shown in Fig. 3. An additional parameter that accounts for internal reflection at the surface of the sample was also included for consistency with previous work $[11,14]$, though the results are essentially independent of this parameter. The stationary solution is plotted in Fig. 5 using the results from the time-resolved measurements with no adjustable parameters. The static and dynamic measurements show that the light propagation in the sample is in excellent agreement with this anisotropic diffusion model. In contrast with a previous result on liquid crystals [6], the static and dynamic measurements of the diffusion tensor agree. The result $D_{x x} / D_{y y}=4.0$ demonstrates the dramatically larger anisotropy in light diffusion in porous gallium phosphide than that measured in liquid crystals $\left(D_{x x} / D_{y y} \sim 1.3\right.$ to $\left.1.6[5,6]\right)$.

There are several intriguing aspects to the results. We have demonstrated optically anisotropic diffusion in a strongly scattering sample for the first time to our knowledge, previous experiments having been performed on weakly scattering and weakly anisotropic nematic liquid crystals. The agreement we find between the static and dynamic measurements seems to contradict the experimental results and theoretical interpretation seen in Ref. [6]. There, it was argued that the diffusion constant, a dynamic quantity, is inaccessible to static measurement and, therefore, that the static and dynamic measurements must probe different quantities. However, the measurements in question probe the ratio of two dynamic quantities, $D_{x x} / D_{y y}$, a value that can be accessed using both dynamic and static probes. This ratio will be determined by both the relative mean free paths (a static property) and by the relative probability to scatter in the $x$ or $y$ direction at each scattering event (the ratio of two dynamic properties). The strong anisotropy in our porous gallium phosphide samples makes this comparison experimentally easier than was possible with liquid crystals. Since the theoretical work has focused exclusively on liquid crystals, the correlation between the microscopic scattering geometry of the samples studied here and the resulting anisotropic diffusive behavior remains an open question. In principal, silicon and other semiconductors could be etched using a similar technique. This would allow for similar experiments to those described here for probing the optical properties of other porous semiconductors. Also, the fact that a porous material can be fabricated between two nonetched layers may allow multilayer etched and nonetched systems to be easily produced. Since the strongly scattering samples described in this Letter have been optimized more for anisotropy than for strong scattering, it is not surprising that localization effects were not observed in comparison with Ref. [10]. Increasing the scattering strength of these samples through chemical etching or other techniques may allow us to ultimately test the effects of anisotropy on localization.

We gratefully acknowledge Jeroen Francke for deposition of the SiN layers. This work is part of the research program of the "Stichting voor Fundamenteel Onderzoek der Materie (FOM)" which is financially supported by the "Nederlandsche Organisatie voor Wetenschappelijk Onderzoek (NWO)."

*Electronic address: P.M.Johnson@phys.uu.nl

${ }^{\dagger}$ Present address: Faculty of Applied Physics and MESA+ Research Institute, University of Twente, 7500 AE Enschede, The Netherlands.

[1] P. Sheng, Introduction to Wave Scattering, Localization, and Mesoscopic Phenomena (Academic, New York, 1995). In the Anderson localization regime, when interference effects become important, diffusive propagation of light can no longer occur. Instead, light intensity has an exponentially decreasing dependence on propagation distance.

[2] A. Yodh and B. Chance, Phys. Today 48, No. 3, 34 (1995).

[3] D. J. Pine, D. A. Weitz, P. M. Chaikin, and E. Herbolzheimer, Phys. Rev. Lett. 60, 1134 (1988).

[4] J. Gomez Rivas, R. Sprik, A. Lagendijk, L. D. Noordam, and C.W. Rella, Phys. Rev. E 63, 046613 (2001); D. S. Wiersma, P. Bartolini, A. Lagendijk, and R. Righini, Nature (London) 390, 671 (1997).

[5] M. H. Kao, K. A. Jester, A. G. Yodh, and P. J. Collings, Phys. Rev. Lett. 77, 2233 (1996).

[6] D. S. Wiersma, A. Muzzi, M. Colocci, and R. Righini, Phys. Rev. Lett. 83, 4321 (1999).

[7] H. Stark and T. C. Lubensky, Phys. Rev. Lett. 77, 2229 (1996).

[8] B. A. van Tiggelen, R. Maynard, and A. Heiderich, Phys. Rev. Lett. 77, 639 (1996).

[9] F. J. P. Schuurmans, D. Vanmaekelbergh, J. van de Lagemaat, and A. Lagendijk, Science 284, 141 (1999); J. Gomez Rivas, R.W. Tjerkstra, D. Vanmaekelbergh, J. J. Kelly, and A. Lagendijk, Appl. Phys. Lett. (to be published).

[10] F. J. P. Schuurmans, M. Megens, D. Vanmaekelbergh, and A. Lagendijk, Phys. Rev. Lett. 83, 2183 (1999).

[11] D. S. Wiersma, A. Muzzi, M. Colocci, and R. Righini, Phys. Rev. E 62, 6681 (2000).

[12] W. Xue, P. Sheng, Q.-J. Chu, and Z.-Q. Zhang, Phys. Rev. Lett. 63, 2837 (1989); Z.-Q. Zhang, Q.-J. Chu, W. Xue, and P. Sheng, Phys. Rev. B 42, 4613 (1990).

[13] R.W. Tjerkstra, J. Gomez Rivas, D. Vanmaekelbergh, and J. J. Kelly, Electrochem. Solid-State Lett. 5, G32 (2002).

[14] J. X. Zhu, D. J. Pine, and D. A. Weitz, Phys. Rev. A 44, 3948 (1991). 